\title{
Numerical Simulation of Water Jet Flow Using Diffusion Flux Mixture Model
}

\author{
Zhi Shang, Jing Lou, and Hongying Li \\ Institute of High Performance Computing (IHPC), Agency for Science, Technology and Research (A*STAR), \\ 1 Fusionopolis Way, No. 16-16 Connexis, Singapore 138632
}

Correspondence should be addressed to Zhi Shang; shangzhi@tsinghua.org.cn

Received 7 May 2014; Revised 24 June 2014; Accepted 30 June 2014; Published 16 July 2014

Academic Editor: Yanzhong Li

Copyright (C) 2014 Zhi Shang et al. This is an open access article distributed under the Creative Commons Attribution License, which permits unrestricted use, distribution, and reproduction in any medium, provided the original work is properly cited.

\begin{abstract}
A multidimensional diffusion flux mixture model was developed to simulate water jet two-phase flows. Through the modification of the gravity using the gradients of the mixture velocity, the centrifugal force on the water droplets was able to be considered. The slip velocities between the continuous phase (gas) and the dispersed phase (water droplets) were able to be calculated through multidimensional diffusion flux velocities based on the modified multidimensional drift flux model. Through the numerical simulations, comparing with the experiments and the simulations of traditional algebraic slip mixture model on the water mist spray, the model was validated.
\end{abstract}

\section{Introduction}

Liquid spray systems are widely used in many chemical, petrochemical, and biochemical industries, such as absorption, oxidation, hydrogenation, coal liquefaction, and aerobic fermentation. The operation of these systems is preferred because of the simple construction, ease of maintenance, and low operating costs. When the spray is injected from nozzle, it causes a turbulent stream to enable an optimum phase exchange. It is built in numerous forms of construction. The mixing is done by the liquid droplets and it requires less energy than mechanical stirring.

A good understanding of the liquid droplet dynamics of the spray will help the engineers to design the high efficient facilities under optimized operating parameters. Although the operation of spray system is simple, due to the complexity of the two-phase turbulent flow, the actual physical flow phenomena are still lacking complete understanding of the fluid dynamics [1].

Many experimental facilities and methods were introduced to study the multiphase flows in spray systems. Ruck and Makiola [2] used a laser Doppler anemometer (LDA) to study the gas-oil droplet passing over a backward facing step. Ferrand et al. [3] used a phase Doppler and laser induced fluorescence technique to study the gas-droplet turbulent velocity and two-phase interaction through a jet with partly responsive droplets. Esposito et al. [4] used a monochrome charge-coupled device CCD camera to study the growth of the droplets. The experimental methods can provide very useful information about the liquid droplets at certain measurement points, but it is difficult for them to show the details of the flow fields and parameters inside the spray.

Following the development of computer technology, it is already allowed to use the numerical method to do the researches in the recent decades [5]. Therefore, many researchers employ the numerical method, called computational fluid dynamics (CFD), to study the details of the flows. Griffiths and Boysan [6] employed the coupled particle Lagrangian model with Eulerian continuous fluid flow model to simulate a cyclone sampler and compared the numerical results with the empirical models. Barton [7] used the stochastic Monte Carlo scheme coupled with $k$ - $\varepsilon$ turbulence model to study the particle trajectories in turbulent flow over a backward facing step. Husted [1] used the EulerianLagrangian model provided by FDS open source software to simulate a water mist spray system for the full spray spreading and compared it with experiments. Through the former studies of CFD method, it can be seen that a good mathematical model will not only help to obtain the agreeable 
TABLE 1: Constants of standard $k$ - $\varepsilon$ turbulence model.

\begin{tabular}{lccccc}
\hline Variable & $C_{\mu}$ & $\sigma_{k}$ & $\sigma_{\varepsilon}$ & $C_{1}$ & $C_{2}$ \\
\hline Constant & 0.09 & 1.0 & 1.3 & 1.44 & 1.92 \\
\hline
\end{tabular}

simulation results but also help to obtain simple, efficient, and accurate ones.

A multidimensional diffusion flux mixture model (DFMM) was developed in this paper. This model was based on the idea of Yang et al. [8] and Shang [9]. It employed a mixture model to describe the multiphase flows based on Eulerian model. The slip velocity can be developed based on the extended 3D drift flux model. Owing to the extension, the effects of gravity and centrifugal force were considered. Through comparisons with experiments and simulations of traditional algebraic slip mixture model (ASMM) on the water mist spray, this model was validated.

\section{Mathematical Modeling}

Considering a problem of turbulent multicomponent multiphase flow with one continuous phase and several dispersed phases, the time average conservation equations of mass, momentum, and energy for the mixture model, the turbulent kinetic energy equation, and the turbulent kinetic energy transport equation can be written as follows:

$$
\begin{aligned}
& \frac{\partial \rho_{m}}{\partial t}+\nabla \cdot\left(\rho_{m} U_{m}\right)=0 \\
& \frac{\partial\left(\rho_{m} U_{m}\right)}{\partial t}+\nabla \cdot\left(\rho_{m} U_{m} U_{m}\right) \\
& =-\nabla p+\rho_{m} g \\
& +\nabla \cdot\left[\left(\mu_{m}+\mu_{t}\right)\left(\nabla U_{m}+\nabla U_{m}^{T}\right)\right] \\
& -\nabla \cdot \sum \alpha_{k} \rho_{k} U_{k m} U_{k m}, \\
& \frac{\partial\left(\rho_{m} h_{m}\right)}{\partial t}+\nabla \cdot\left(\rho_{m} U_{m} h_{m}\right) \\
& =q+\nabla \cdot\left[\left(\frac{\mu_{m}}{\operatorname{Pr}}+\frac{\mu_{t}}{\operatorname{Pr}_{t}}\right) \nabla h_{m}\right]-\nabla \cdot \sum \alpha_{k} \rho_{k} h_{k} U_{k m}, \\
& \frac{\partial\left(\rho_{m} k\right)}{\partial t}+\nabla \cdot\left(\rho_{m} U_{m} k\right) \\
& =\nabla \cdot\left[\left(\mu_{m}+\frac{\mu_{t}}{\sigma_{k}}\right) \nabla k\right]+G-\rho_{m} \varepsilon, \\
& \frac{\partial\left(\rho_{m} \varepsilon\right)}{\partial t}+\nabla \cdot\left(\rho_{m} U_{m} \varepsilon\right) \\
& =\nabla \cdot\left[\left(\mu_{m}+\frac{\mu_{t}}{\sigma_{\varepsilon}}\right) \nabla \varepsilon\right]+\frac{\varepsilon}{k}\left(C_{1} G-C_{2} \rho_{m} \varepsilon\right),
\end{aligned}
$$

in which

$$
\begin{aligned}
& \rho_{m}=\sum \alpha_{k} \rho_{k}, \\
& \mu_{m}=\sum \alpha_{k} \mu_{k}, \\
& \rho_{m} U_{m}=\sum \alpha_{k} \rho_{k} U_{k}, \\
& U_{k m}=U_{k}-U_{m}, \\
& G=\frac{1}{2} \mu_{t}\left[\nabla U_{m}+\left(\nabla U_{m}\right)^{T}\right]^{2}, \\
& \mu_{t}=C_{\mu} \rho_{m} \frac{k^{2}}{\varepsilon},
\end{aligned}
$$

where $\rho$ is the density, $U$ is the velocity vector, $\alpha$ is the volumetric fraction, $p$ is pressure, $g$ is the gravitational acceleration vector, $U_{k m}$ is the diffusion velocity vector of $k$ dispersed phase relative to the averaged mixture flow, $h$ is enthalpy, $q$ is heat input, $\mu$ is viscosity, $\mu_{t}$ is turbulent viscosity, $\mathrm{Pr}$ is molecular Prandtl number, $\operatorname{Pr}_{t}$ is turbulent Prandtl number, and $G$ is stress production. $C_{\mu}, \sigma_{k}, \sigma_{\varepsilon}, C_{1}, C_{2}$ are constants for standard $k-\varepsilon$ turbulence model [10], shown in Table 1 . The subscript $m$ stands for the averaged mixture flow, and $k$ stands for $k$ dispersed phase.

Additional to the above equations, the following conservation equation for each dispersed phase is also necessary.

Consider the following:

$$
\frac{\partial\left(\alpha_{k} \rho_{k}\right)}{\partial t}+\nabla \cdot\left(\alpha_{k} \rho_{k} U_{m}\right)=\Gamma_{k}-\nabla \cdot\left(\alpha_{k} \rho_{k} U_{k m}\right),
$$

where $\Gamma_{k}$ is the generation rate of $k$-phase.

In order to closure the governing equations (1) (12), it is necessary to determine the diffusion velocities $U_{\mathrm{km}}$. The following equation is employed to covert the diffusion velocities to slip velocities that can be presented as $U_{k c}=$ $U_{k}-U_{c}$, where $U_{c}$ is the velocity of the continuous phase.

Consider the following:

$$
U_{k m}=U_{k c}-\sum \frac{\alpha_{k} \rho_{k}}{\rho_{m}} U_{k c} .
$$

Actually the above equation can be developed from the definition of the mixture density equation (6), the definition of mixture mass flux equation (8), and the diffusion velocity equation (9). Once the slip velocities are obtained, the whole governing equations will be closured.

Because the slip velocities present the difference of the movement between the dispersed phase, for instance, the liquid droplet, and the continuous phase, for instance, the gas, in gas liquid-droplet two-phase flow, the slip velocity can be modeled through the drift flux model [8], shown in (14):

$$
\begin{gathered}
U_{l g}=-1.53\left[\frac{\sigma\left(\rho_{l}-\rho_{g}\right) g^{\prime}}{\rho_{g}^{2}}\right]^{1 / 4} \frac{g^{\prime}}{\left|g^{\prime}\right|}, \\
g^{\prime}=g-\frac{d U_{f}}{d t}=g-\left(\frac{\partial U_{f}}{\partial t}+U_{f} \cdot \nabla U_{f}\right), \\
U_{f}=\alpha_{g} U_{g}+\alpha_{l} U_{l},
\end{gathered}
$$




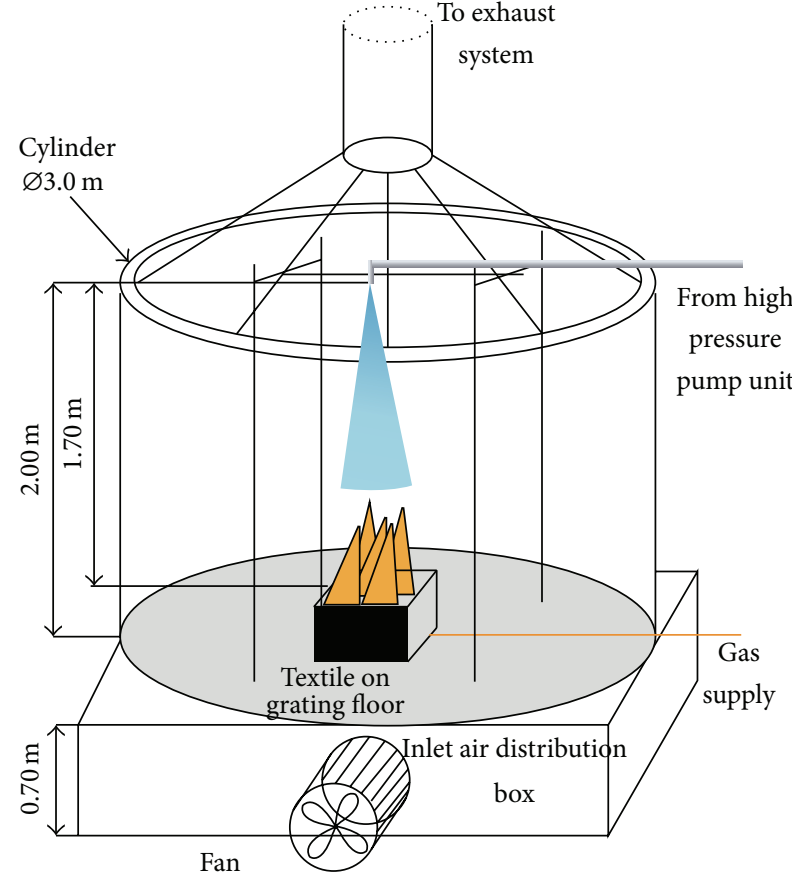

(a) Sketch

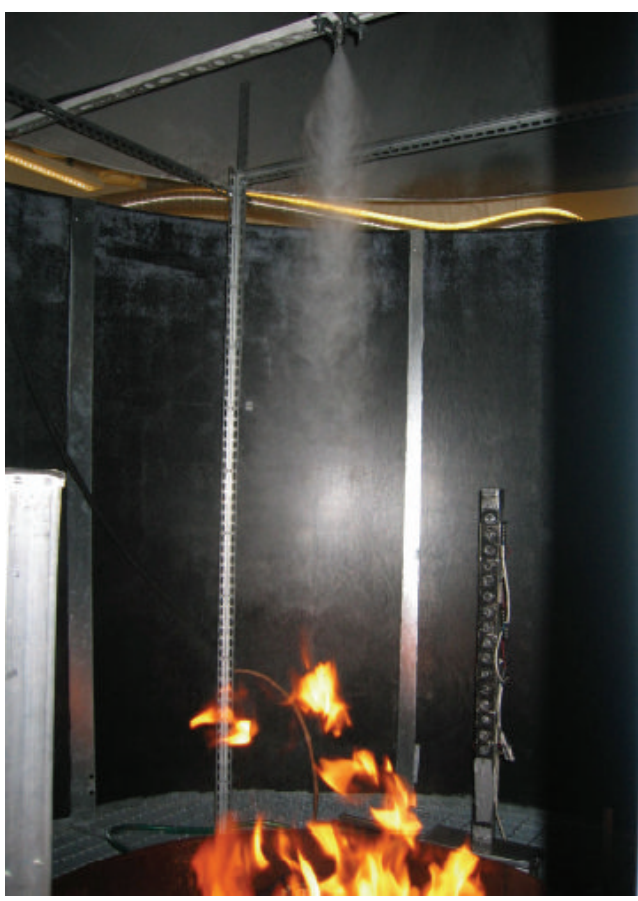

(b) Experiment

FIGURE 1: Diagrammatic sketch of the experiment facility [1].

where $U_{l g}$ is the slip velocity between liquid droplet and gas, $\sigma$ is surface tension, $g$ is gravity, and $U_{f}$ is drift velocity.

In (14), the drift flux model is different from the traditional 1D drift flux model in Zuber and Findlay [11] and in Hibiki and Ishii [12]. It adopts the form as Yang et al. [8] and Shang [9]. In Yang et al. [8], the centrifugal force was induced by the mixture volumetric flux to revise the gravity considering the natural curve movement of liquid droplets. Owing to the concerns, the traditional 1D drift flux model $[11,12]$ was extended to $3 \mathrm{D}$. Because of being extended, the revised 3D drift flux model is able to describe complex flow conditions and to be adopted by CFD. Further, in this paper, the gradient of mixture velocity, which is used to calculate the centrifugal force, is innovatively induced to revise and update Yang et al.s [8] 3D drift flux model. After the updating, the new terminal velocity model, shown in (14), (15), and (16), is able to suit the gas liquid-droplet two-phase flows. Since the slip velocity is determined, the whole equations are closured to be solved.

\section{Numerical Procedures}

In the simulations, the CFD technique was based on ANSYS FLUENT 13.0. In ANSYS FLUENT, during the numerical computing, all differential governing equations are solved by applying a finite volumes method (FVM). For the fluids, the spatial discretization was performed by upwind scheme of second order for all conservation equations and phase coupled SIMPLE scheme was used for coupling between the pressure and velocity. The overall spatial discretization was of the second-order accuracy. The first order implicit scheme was used for the time discretization. During the numerical simulations, the pressure-based solver was employed because all the gas and liquid phases were considered as incompressible fluids. The fixed time stepping method was employed to run the transient simulations.

The terminal velocity model was accomplished using the concept of user defined functions (UDF). This numerical solution was implemented as a subroutine and linked to the ANSYS FLUENT solver via a set of the original UDFs. The decision whether the UDFs of the coefficients should be used for the interfacial forces at the given calculation step was made automatically by the solver.

\section{Numerical Simulations}

Husted [1] did the experiment on a water mist spray system using a particle image velocimetry (PIV), a phase Doppler anemometer (PDA), and a high-speed camera measurement technology. Figure 1 shows the diagrammatic sketch of facility of the experiment. In Husted's [1] experiment, the water mist spray sprayed down from the top nozzle is used for fire extinguishing. The fire can be set at the extruded square at the bottom. If there is no fire, the extruded square can be treated as a solid wall. The purpose of the experiment was to fill the lack of guidelines available for dimensioning water mist systems. The experiment data were good to be used to measure the modeling and numerical simulations. Therefore this experiment was chosen in this paper as the validation tool to validate the drift flux mixture model.

According to the experiment, Figure 2 shows the simplified geometry and the mesh generation for CFD applications. 


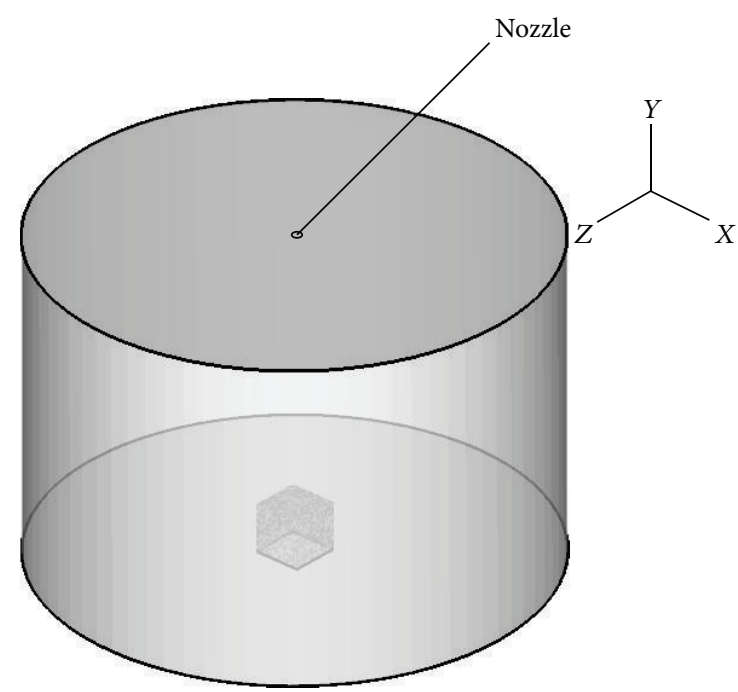

(a) Geometry

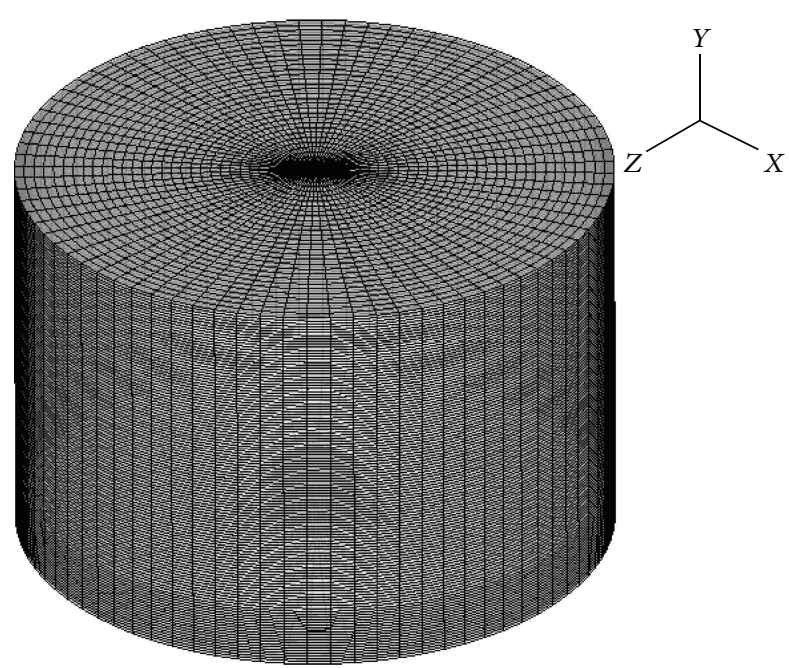

(b) Mesh

FIGURE 2: Simplified geometry and mesh generation.

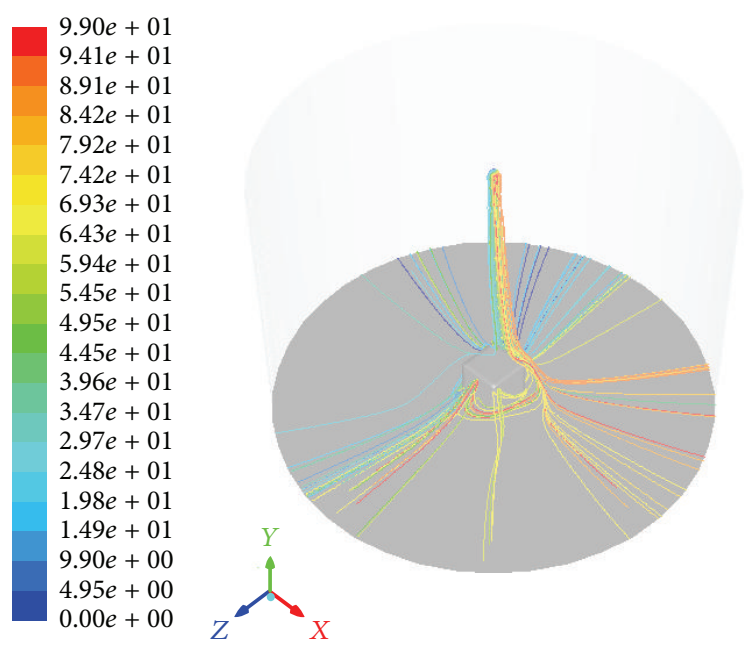

FIgURE 3: Tracked path lines of the water droplets.

The water nozzle is just a simple round hole at the top of the geometry. Due to the fact that the experiment [1] has only the data of the distributions of velocities without considering the heated situation, the simulations are only for the velocity same as the experiment without considering the thermal equation (3) [1].

During the simulations, the numerical exercise was performed in a three-dimensional (3D) environment. All the parameters used were similar to those by Husted [1]. The water droplets are sprayed from the top nozzle with flow rate of 0.38 liter per minute. The bottom is solid wall and the other sides are opened to the environment. Before the formal simulations, the mesh independency studies were performed and it was found that the mesh size of 328000 was enough for the water spray case for guaranteeing the simulation results being mesh-independent. During the simulations, the time step was set as a constant of 0.01 seconds which is quick enough for the simulations because the simulation time for every case lasted long enough until its steady state.

Figure 3 shows the tracked path lines of the water droplets. From Figure 3, it can be seen that the droplets flow down in the middle and gradually spread like a cone shape down from the nozzle. According to the studies of Husted [1], the stages of the development of the cone spray can be separated as initial conical zone, inflow zone, transition zone, turbulent zone, and full cone zone. When the distance is far from $500 \mathrm{~mm}$ down from the nozzle, the spray is fully mixed and drop distribution is uniform. The drop velocity becomes flat.

Figure 4 shows the comparisons of the water mist droplet velocity of the simulations with experiments along different positions down from the nozzle. In Figure 4, the reference numerical simulations were carried out by the traditional algebraic slip mixture model in FLUENT. The SchillerNaumann drag force model [13] was adopted during the simulations for both of the diffusion flux model and the traditional algebraic slip mixture model [14] due to the fact that the turbulent dispersion force was considered.

From the comparisons, it can be seen that the diffusion flux mixture model is able to capture the velocity profile the same as experiments. The numerical predictions not only have the peak value quite close to the experiment but also have the position approaching to the experiment. The results are much better than the predictions by traditional algebraic slip mixture model in FLUENT.

\section{Conclusion}

The novel multidimensional diffusion flux mixture model is developed based on the mixture multiphase flow model. The diffusion velocity between the dispersed phase and the mixture is closured through the slip velocity. The slip velocity 


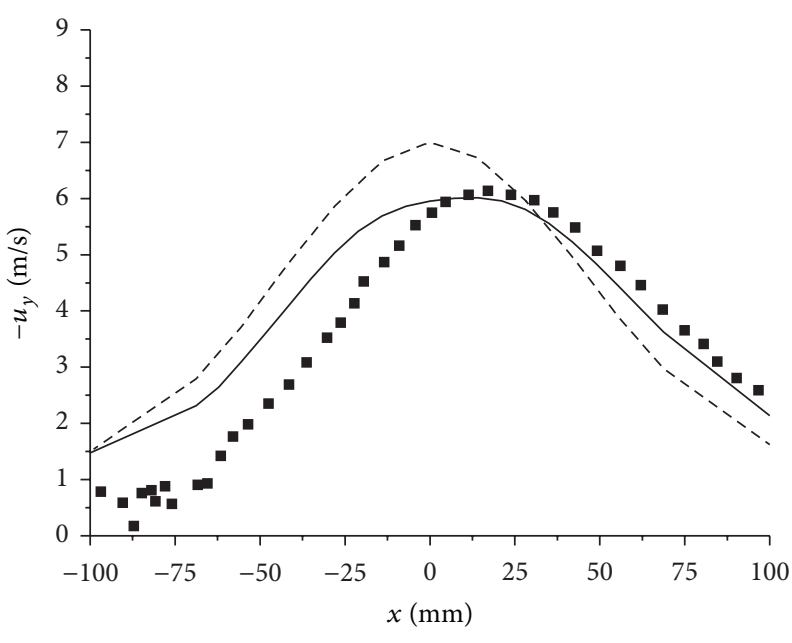

(a) $y=550 \mathrm{~mm}$ down from nozzle

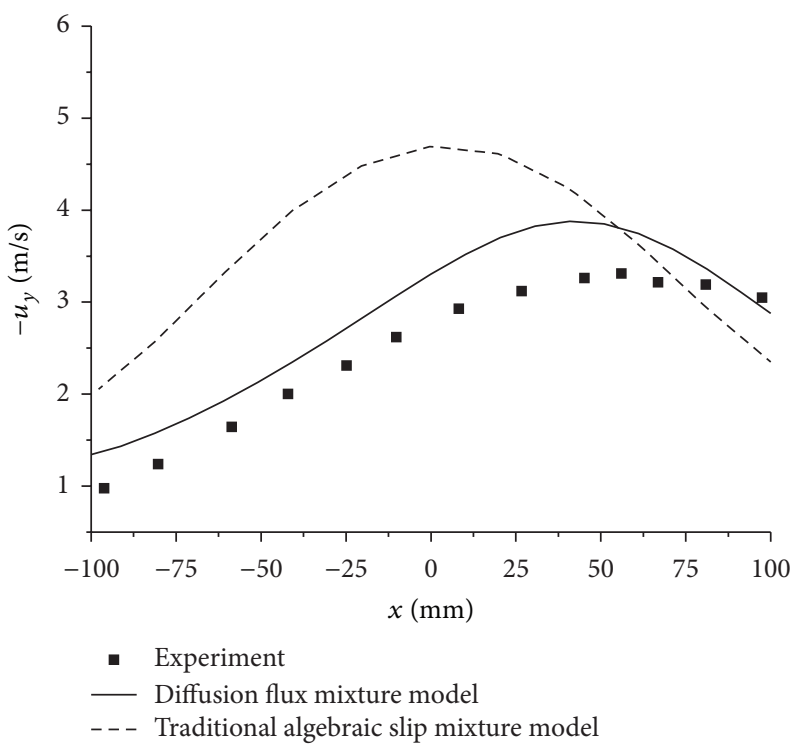

(c) $y=1010 \mathrm{~mm}$ down from nozzle

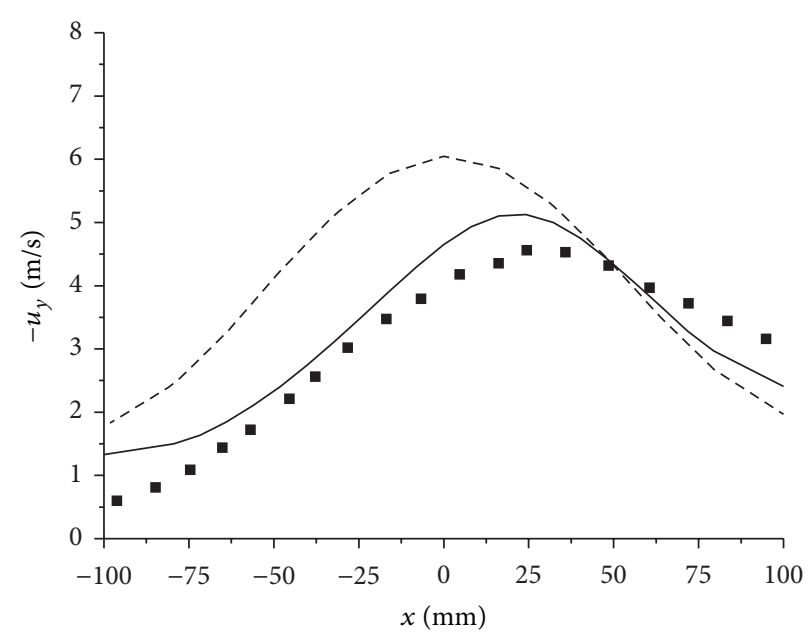

(b) $y=700 \mathrm{~mm}$ down from nozzle

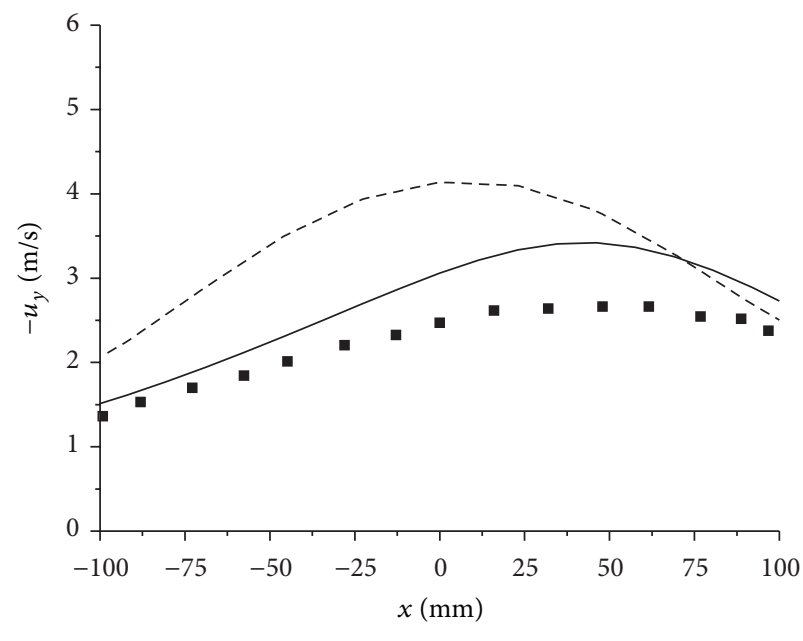

- Experiment

— Diffusion flux mixture model

- - - Traditional algebraic slip mixture model

(d) $y=1200 \mathrm{~mm}$ down from nozzle

Figure 4: Comparisons of water mist droplet velocity under different positions down from nozzle.

is developed through the drift flux model. Accordingly, the multidimensional diffusion flux mixture model is different from the traditional algebraic slip mixture model. Through the comparisons of the numerical simulations between the multidimensional diffusion flux mixture model and experiments, the model is validated. Through comparisons of the numerical simulations between the multidimensional diffusion flux mixture model and the numerical simulations of the traditional algebraic slip mixture model, the efficiency and accuracy of the model are confirmed.

\section{Conflict of Interests}

The authors declare that there is no conflict of interests regarding the publication of this paper.

\section{Acknowledgment}

The authors would like to acknowledge the support provided by Multiphase Flow for Deep-Sea Oil \& Gas DownHole Applications - SERC TSRP Programme of Agency for Science, Technology and Research ( $\left.\mathrm{A}^{*} \mathrm{STAR}\right)$ in Singapore (Reference no. 102164 0075).

\section{References}

[1] B. P. Husted, Experimental measurements of water mist systems and implications for modeling in CFD [Ph.D. thesis], Lund University, Lund, Sweden, 2007.

[2] B. Ruck and B. Makiola, "Particle dispersion in a single-sided backward-facing step flow," International Journal of Multiphase Flow, vol. 14, no. 6, pp. 787-800, 1988.

[3] V. Ferrand, R. Bazile, J. Borée, and G. Charnay, "Gas-droplet turbulent velocity correlations and two-phase interaction in 
an axisymmetric jet laden with partly responsive droplets," International Journal of Multiphase Flow, vol. 29, no. 2, pp. 195217, 2003.

[4] A. Esposito, A. D. Montello, Y. G. Guezennec, and C. Pianese, "Experimental investigation of water droplet-air flow interaction in a non-reacting PEM fuel cell channel," Journal of Power Sources, vol. 195, no. 9, pp. 2691-2699, 2010.

[5] Z. Shang, Y. Yao, and S. Chen, "Numerical investigation of system pressure effect on heat transfer of supercritical water flows in a horizontal round tube," Chemical Engineering Science, vol. 63, no. 16, pp. 4150-4158, 2008.

[6] W. D. Griffiths and F. Boysan, "Computational fluid dynamics (CFD) and empirical modelling of the performance of a number of cyclone samplers," Journal of Aerosol Science, vol. 27, no. 2, pp. 281-304, 1996.

[7] I. Barton, "Simulation of particle trajectories in turbulent flow over a backward-facing step," $R$ \& D Journal, vol. 15, no. 3, pp. 65-78, 1999.

[8] R. Yang, R. Zheng, and Y. Wang, "Analysis of two-dimensional two-phase flow in horizontal heated tube bundles using drift flux model," Heat and Mass Transfer, vol. 35, no. 1, pp. 81-88, 1999.

[9] Z. Shang, "CFD of turbulent transport of particles behind a backward-facing step using a new model-k- $\varepsilon$-Sp," Applied Mathematical Modelling, vol. 29, no. 9, pp. 885-901, 2005.

[10] B. E. Launder and D. B. Spalding, "The numerical computation of turbulent flows," Computer Methods in Applied Mechanics and Engineering, vol. 3, no. 2, pp. 269-289, 1974.

[11] N. Zuber and J. A. Findlay, "The effects of non-uniform flow and concentration distributions and the effect of the local relative velocity on the average volumetric concentration in two-phase flow," Tech. Rep. GEAP-4592, 1964.

[12] T. Hibiki and M. Ishii, "Distribution parameter and drift velocity of drift-flux model in bubbly flow," International Journal of Heat and Mass Transfer, vol. 45, no. 4, pp. 707-721, 2002.

[13] L. Schiller and Z. Z. Naumann, "Über die grundlegenden Berechungen bei der schwerkraftaufbereitung," Zeitschrift des Vereines Deutscher Ingenieure, vol. 77, pp. 318-325, 1935.

[14] O. Simonin and P. L. Viollet, "Modeling of turbulent two-phase jets loaded with discrete particles," in Phenomena in Multiphase Flows, pp. 259-269, 1990. 

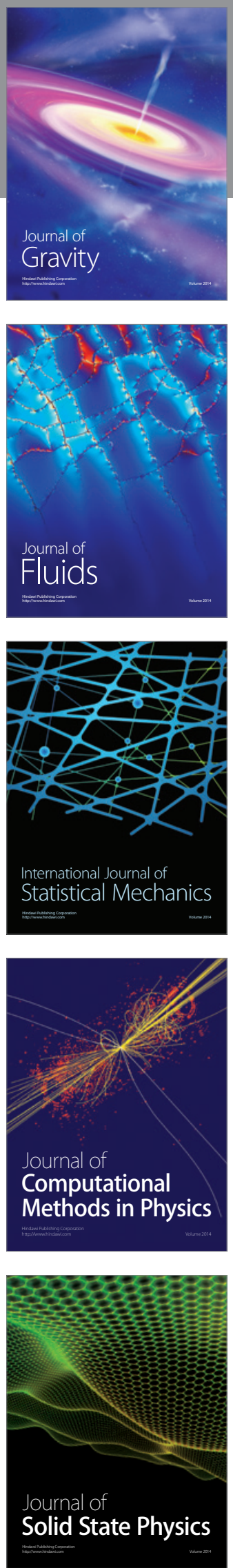

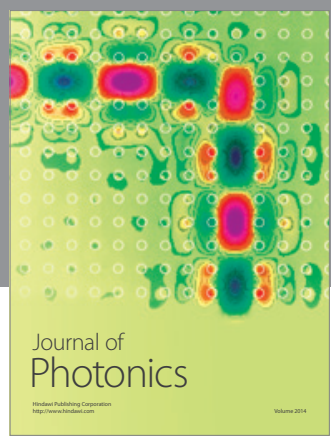

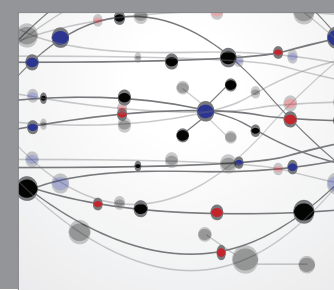

The Scientific World Journal

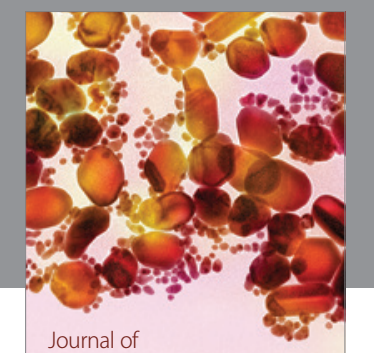

Soft Matter
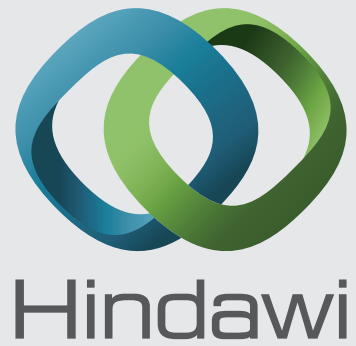

Submit your manuscripts at

http://www.hindawi.com
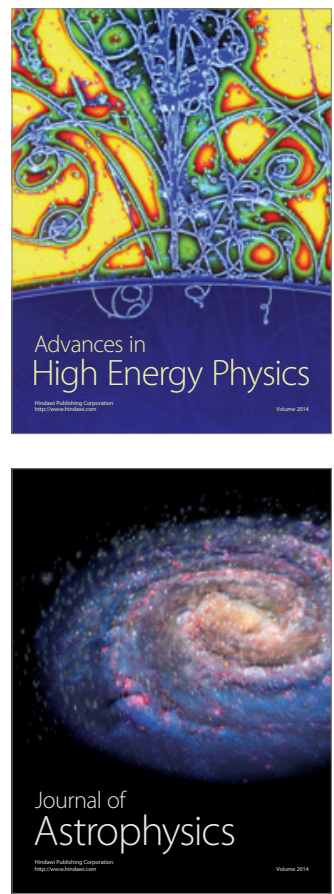
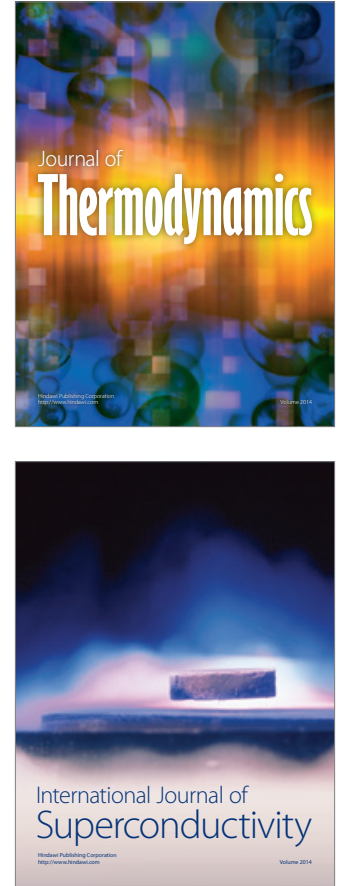
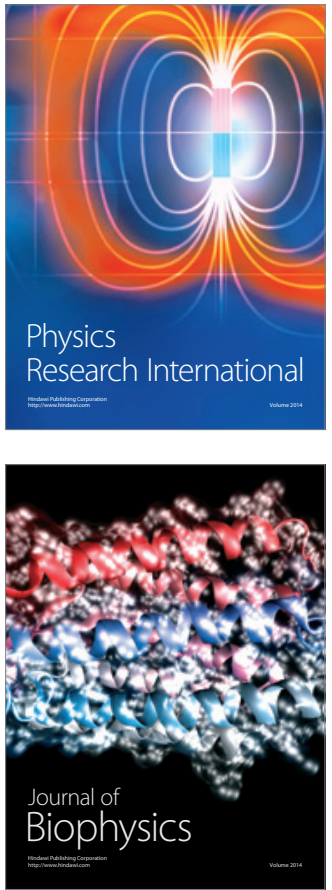
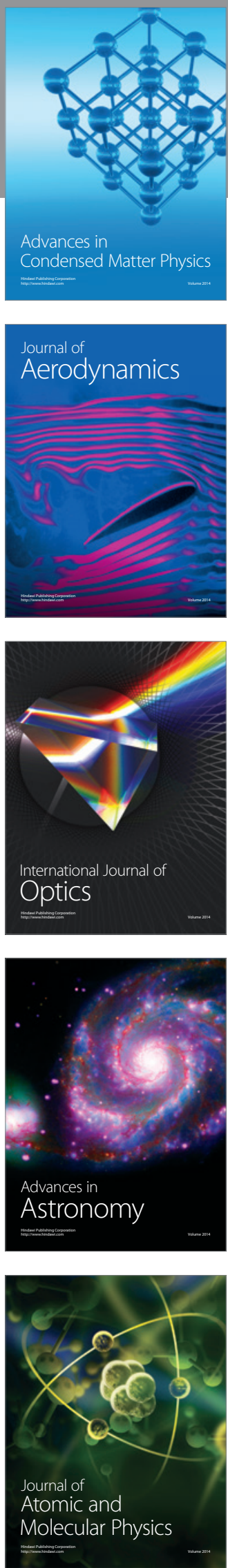